\title{
Passenger Traffic Evaluation and Price Formation on the Transportation Services Market
}

\author{
V. M. Bure, ${ }^{1}$ V. V. Mazalov, ${ }^{2}$ A. V. Melnik, ${ }^{1}$ and N. V. Plaksina ${ }^{3}$ \\ ${ }^{1}$ Saint Petersburg State University, Saint Petersburg 198504, Russia \\ ${ }^{2}$ Institute of Applied Mathematical Research, Karelian Research Center, Russian Academy of Sciences, Petrozavodsk 185910, Russia \\ ${ }^{3}$ Petrozavodsk State University, Petrozavodsk 185910, Russia
}

Correspondence should be addressed to A. V. Melnik; annamazalova@yandex.ru

Received 6 October 2014; Accepted 17 January 2015

Academic Editor: Ahmed Ghoniem

Copyright (C) 2015 V. M. Bure et al. This is an open access article distributed under the Creative Commons Attribution License, which permits unrestricted use, distribution, and reproduction in any medium, provided the original work is properly cited.

\begin{abstract}
This paper investigates equilibrium formation in the passenger traffic model. First, we propose an estimation technique for the distribution of incoming passengers at each stop with respect to subsequent stops of a route based on available information on incoming and outgoing passengers. Second, we employ the obtained information on passenger traffic to introduce a game-theoretic model of passenger traffic distribution with respect to transport facilities.
\end{abstract}

\section{Introduction}

The rapid expansion of megapolises and the growing number of private vehicles pose the problem of passenger traffic control. We comprehend a passenger traffic flow as the intensity of passenger traffic on a certain route. Passenger traffic flows play an important role in defining the routes of urban transport and optimal traffic intervals of public transport facilities. Passenger traffic flows are nonuniform, that is, possess time-to-time variability (by hours, circadian periods, days of week, and seasons). The passenger traffic capacity of a separate district is determined depending on the number of residents, their mobility indices, and the temporal nonuniformity coefficients of traffic.

Different analysis techniques serve for acquiring information on the mobility indices of residents and the temporal nonuniformity coefficients of their traffic. Natural experiments guarantee higher accuracy; the error of such methods constitutes approximately $5 \%$. Their major drawback consists in appreciable monetary and human resources required; data treatment consumes much time. Therefore, the results of natural observations may provide an inaccurate picture of real passenger traffic. Another approach to passenger traffic evaluation employs gravitation- and entropy-based methods (Wilson [1-3]). Within the framework of the entropy model, passenger traffic flows are calculated via demographic and socioeconomic data with information on the actual location of a district. The main disadvantage of the entropy-based method is the stationarity of passenger traffic flows. And so, natural experiments and demographic data are often combined with statistical methods and queues theory methods to obtain up-to-date information on passenger traffic (Draper and Smith [4], Taha [5], and Sheffy [6]). In the first part of this paper, we propose a statistical evaluation method for the intensities of passenger traffic flows using information on the incoming and outgoing passengers at different stops. Similar methods seem reasonable for constructing equilibrium solutions in traffic planning by acquired information on passenger traffic flows.

Recently, much attention has been paid to statements taking into account the behavioral features of the transport model participants, namely, passengers and carriers (transport companies). Among pioneering results, we mention the Wardrop model on the equilibrium distribution of transport flows depending on the capacity of a route (Wardrop [7]). According to the Wardrop principle, the trip time along all existing routes is the same for all road users and smaller than the trip time of any road user in the case of its route diversion. Moreover, the average trip time gets minimized. The mathematical model suggested by Wardrop became 
classical; nowadays, it represents a basic tool in the theory of traffic flows (Spiess and Florian [8] and Yang and Huang [9]).

In addition to this direction of road traffic research, queueing theory also yielded a series of works analyzing the flows of active clients. Here clients choose a preferable service from some viewpoint and corresponding service queues get formed. This considerably changes the dynamics of queues and classic methods of queueing theory appear inapplicable. Under the hypothesis of users rational behavior, the authors Naor [10], Luski [11], Levhari and Luski [12], Chen and Wan [13], Hotelling [14], and Hassin and Haviv [15] found the equilibrium value of service flows. The effect of pricing on queue for the monopoly case is first studied in [10]. In papers [11-13], the duopoly has been considered in price-and-time competition. The authors utilize the idea of Hotelling's model on the rational behavior of users [14]. They consider a market with Poisson arrivals to two firms with exponential service times. By assuming that passengers are distributed with respect to services according to their cost functions (the price of service plus service time), they introduce a game-theoretic model with service centers acting as players. An extensive survey on pricing with queues can be found in [15]. In the present paper, we continue this approach for the analysis of pricing game in the general transportation network with several firms, as well as evaluating an equilibrium in a series of cases.

\section{Characteristics Evaluation for Passenger Traffic Flows}

In the general case, the transport model can be represented by a graph whose nodes act as stops and edges describe transport passageways. Choose a certain route of a transport vehicle, that is, a sequence of $K$ stops connected by edges (see Figure 1). Consider the passenger traffic flow on this route. Let $x_{1}^{r}, x_{2}^{r}, \ldots, x_{K}^{r}$ be the numbers of incoming passengers at stops $i=1, \ldots, K$, respectively, in experiment $r, r=1, \ldots, N$. By analogy, the quantities $y_{1}^{r}, y_{2}^{r}, \ldots, y_{K}^{r}$ will characterize the number of outgoing passengers at stops $j=1, \ldots, K$, respectively, in experiment $r, r=1, \ldots, N$. Obviously, $x_{K}^{r}=$ $y_{1}^{r}=0$ for any experiment $r$. By assumption, $x_{i}^{r}$ and $y_{j}^{r}$ form observable quantities in the model. Denote by $\lambda_{i j}^{r}$ the number of passengers incoming at stop $i$ and outgoing at stop $j$. Actually, these are unobservable quantities to be estimated. Exactly their average value gives the intensity of passenger traffic flow from point $i$ to point $j$. For convenience, information on passenger traffic flows is often combined in the tables; see Table 1.

As a matter of fact, Table 1 demonstrates the results of experiment $r$. All elements in the last column are the sums of elements from a corresponding row; similarly, the last row contains the sums of elements from a corresponding column. The quantity $\lambda_{i j}^{r}$ indicates the (unobservable) number of passengers incoming at stop $i$ and outgoing at stop $j$ in experiment $r$ :

$$
y_{j}^{r}=\sum_{i=1}^{j-1} \lambda_{i j}^{r} .
$$

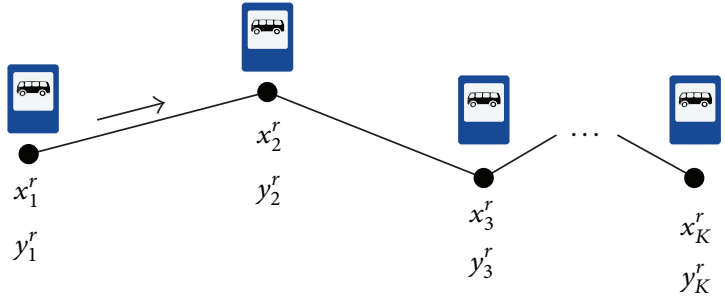

Figure 1: Route of $K$ stops.

TABLE 1: Information on passenger traffic flow.

\begin{tabular}{lcccccc}
\hline$\lambda_{11}^{r}=0$ & $\lambda_{12}^{r}$ & $\lambda_{13}^{r}$ & $\cdots$ & $\lambda_{1 K-1}^{r}$ & $\lambda_{1 K}^{r}$ & $x_{1}^{r}$ \\
\hline 0 & $\lambda_{22}^{r}=0$ & $\lambda_{23}^{r}$ & $\cdots$ & $\lambda_{2 K-2}^{r}$ & $\lambda_{2 K}^{r}$ & $x_{2}^{r}$ \\
\hline$\vdots$ & $\vdots$ & $\vdots$ & $\vdots$ & $\vdots$ & $\vdots$ & $\vdots$ \\
\hline 0 & 0 & 0 & $\cdots$ & $\lambda_{K-1, K-1}^{r}=0$ & $\lambda_{K-1, K}^{r}$ & $x_{K-1}^{r}$ \\
\hline 0 & 0 & 0 & $\cdots$ & 0 & 0 & $x_{K}^{r}=0$ \\
\hline$y_{1}^{r}=0$ & $y_{2}^{r}$ & $y_{3}^{r}$ & $\cdots$ & $y_{K-1}^{r}$ & $y_{K}^{r}$ & \\
\hline
\end{tabular}

TABLE 2: Information on the probabilistic distribution of passengers.

\begin{tabular}{ccccccc}
\hline$p_{11}=0$ & $p_{12}$ & $p_{13}$ & $\cdots$ & $p_{1 K-1}$ & $p_{1 K}$ & 1 \\
\hline 0 & $p_{22}=0$ & $p_{23}$ & $\cdots$ & $p_{2 K-1}$ & $p_{2 K}$ & 1 \\
\hline$\vdots$ & $\vdots$ & $\vdots$ & $\vdots$ & $\vdots$ & $\vdots$ & $\vdots$ \\
\hline 0 & 0 & 0 & $\cdots$ & $p_{K-1, K-1}=0$ & $p_{K-1, K}$ & 1 \\
\hline
\end{tabular}

In each experiment, we observe the elements of the last column and row. For all $i$ and $j$, suppose that the passenger traffic flows $\lambda_{i j}^{r}$ represent independent (for different $r$ ) identically distributed random quantities. Their average value is the intensity of passenger traffic over an appropriate route. Designate by $p_{i j}$ the share of passengers incoming at stop $i$ and outgoing at stop $j$. It can be interpreted as the probability that a passenger entering at stop $i$ leaves at stop $j$. Construct Table 2 from these values.

In Table 2, the sum of elements in each row equals 1 ; interestingly, this is the polynomial distribution of the probabilities of going out for passengers incoming at an appropriate stop (whose number coincides with the index of row).

Consider experiment $r$ and multiply row $i$ from Table 2 by $x_{i}^{r}$. Naturally, the resulting row corresponds to row $i$ from Table 1 , since $x_{i}^{r} p_{i j}$ is the expected value of the random quantity $\lambda_{i j}^{r}$. Moreover, random quantities in the same column of Table 1 appear to be mutually independent. Hence, their deviations from the expected value are random and independent and compensate each other in case of summation. Therefore, we have the following equality in a rough approximation:

$$
y_{j}^{r} \approx \sum_{i=1}^{j-1} x_{i}^{r} p_{i j} .
$$

If the number of experiments $N$ is large, (2) often enjoy linear independence (the coefficients in different equations form 
mutually independent random quantities). Consequently, these equations can be resolved in the unknown probabilities $p_{i j}$ (here $i<j, j=1, \ldots, K$ ) by applying the least-squares technique (see for instance, Greene [16]) or the minimization method for the sum of absolute deviations (Davino et al. [17]).

In the former case, the problem consists in minimizing the sum of squared deviations

$$
s=\sum_{r=1}^{N} \sum_{j=2}^{K}\left(y_{j}^{r}-\sum_{i=1}^{j-1} x_{i}^{r} p_{i j}\right)^{2},
$$

subject to the constraints

$$
\begin{gathered}
\sum_{j=i+1}^{K} p_{i j}=1, \quad i=1, \ldots, K-1, \\
p_{i j} \geq 0, \quad \forall i, j .
\end{gathered}
$$

In the latter case, the desired values $p_{i j}$ minimize the functional

$$
s_{1}=\sum_{r=1}^{N} \sum_{j=2}^{K}\left|y_{j}^{r}-\sum_{i=1}^{j-1} x_{i}^{r} p_{i j}\right|,
$$

subject to the same constraints.

The first problem can be easily solved by Lagrange's method of multipliers. Let us focus on the second optimization problem and show its solution by linear programming methods.

Introduce the new variables:

$$
\begin{aligned}
& Z_{j}^{r}= \begin{cases}y_{j}^{r}-\sum_{i=1}^{j-1} x_{i}^{r} p_{i j}, & \text { if } y_{j}^{r}-\sum_{i=1}^{j-1} x_{i}^{r} p_{i j} \geq 0, \\
0, & \text { if } y_{j}^{r}-\sum_{i=1}^{j-1} x_{i}^{r} p_{i j}<0,\end{cases} \\
& W_{j}^{r}= \begin{cases}\sum_{i=1}^{j-1} x_{i}^{r} p_{i j}-y_{j}^{r}, & \text { if } y_{j}^{r}-\sum_{i=1}^{j-1} x_{i}^{r} p_{i j} \leq 0, \\
0, & \text { if } y_{j}^{r}-\sum_{i=1}^{j-1} x_{i}^{r} p_{i j}>0 .\end{cases}
\end{aligned}
$$

Clearly,

$$
\begin{gathered}
y_{j}^{r}-\sum_{i=1}^{j-1} x_{i}^{r} p_{i j}=Z_{j}^{r}-W_{j}^{r}, \\
\left|y_{j}^{r}-\sum_{i=1}^{j-1} x_{i}^{r} p_{i j}\right|=Z_{j}^{r}+W_{j}^{r} .
\end{gathered}
$$

Then, the problem is to minimize the function:

$$
s_{1}=\sum_{r=1}^{N} \sum_{j=2}^{K}\left(Z_{j}^{r}+W_{j}^{r}\right),
$$

subject to the constraints

$$
\begin{gathered}
\sum_{j=i+1}^{K-1} p_{i j}=1, \quad \forall i ; \\
y_{j}^{r}=\sum_{i=1}^{j-1} x_{i}^{r} p_{i j}+Z_{j}^{r}-W_{j}^{r}, \quad j=2, \ldots, K ; \\
Z_{j}^{r} \geq 0, \quad W_{j}^{r} \geq 0, \quad r=1, \ldots, N ; \\
p_{i j} \geq 0, \quad \forall i, j .
\end{gathered}
$$

Here the unknown variables are the quantities $p_{i j}, Z_{j}^{r}$, and $W_{j}^{r}(i<j \leq K, i=1, \ldots, K-1$ and $r=1, \ldots, N)$.

Consider an experiment with a route containing five stops. Let us analyze passenger traffic flows on this route. According to the model, $p_{12}$ reflects the passenger traffic flow from stop 1 to stop 2, $p_{13}$ describes the passenger traffic flow from stop 1 to stop 3, and so on. In the experiment, the number of incoming passengers is a random variable with the following distribution: the number of incoming passengers on first stop can be 4,5 , or 6 with equal probabilities $1 / 3$; on second and fourth stops, this value is 2 or 3 with probabilities $1 / 2$; and on third stop, it equals 1 or 2 with probabilities $1 / 2$. Outgoing passengers in each stop are equal to the number of passengers at the vehicle minus a random variable which takes values 0,1 , or 2 with probabilities $1 / 3$. For evaluating the characteristics of passenger traffic flows, we have conducted 100 numerical experiments and compiled the following tables of passenger traffic.

Tables 3-4 present the information on passenger traffic flows for first 15 experiments.

To find the unknown variables $p_{i j}(i<j, j=1, \ldots, K)$, employ the least-squares technique. The functional (3) gets minimized under the following values of the parameters: $p_{12}=0.48 ; p_{13}=0.52 ; p_{14}=0 ; p_{15}=0 ; p_{23}=0.67 ;$ $p_{24}=0.33 ; p_{25}=0 ; p_{34}=1 ; p_{35}=0 ; p_{45}=1$.

Minimization of the absolute values of the deviations (5) by the simplex method has yielded the following results: $p_{12}=$ $0.5 ; p_{13}=0.49 ; p_{14}=0.01 ; p_{15}=0 ; p_{23}=0.68 ; p_{24}=0.32$; $p_{25}=0 ; p_{34}=1 ; p_{35}=0 ; p_{45}=1$.

These numerical results lead to the passenger traffic intensity matrices:

$$
\begin{aligned}
& \Lambda_{1}=\left(\begin{array}{ccccc}
0 & 2.5 & 2.45 & 0.05 & 0 \\
0 & 0 & 2.04 & 0.96 & 0 \\
0 & 0 & 0 & 1 & 0 \\
0 & 0 & 0 & 0 & 2 \\
0 & 0 & 0 & 0 & 0
\end{array}\right), \\
& \Lambda_{2}=\left(\begin{array}{ccccc}
0 & 2.4 & 2.6 & 0 & 0 \\
0 & 0 & 2.01 & 0.99 & 0 \\
0 & 0 & 0 & 1 & 0 \\
0 & 0 & 0 & 0 & 2 \\
0 & 0 & 0 & 0 & 0
\end{array}\right),
\end{aligned}
$$

for problems (3) and (5), respectively.

The accumulated information on passenger traffic flows allows making certain conclusions on the volumes of passenger traffic flows for different directions. The largest passenger 
TABLE 3: Information on the number of incoming passengers.

\begin{tabular}{|c|c|c|c|c|c|c|c|c|c|c|c|c|c|c|c|}
\hline \multirow{2}{*}{ Stop number } & \multicolumn{15}{|c|}{ Experiment number } \\
\hline & 1 & 2 & 3 & 4 & 5 & 6 & 7 & 8 & 9 & 10 & 11 & 12 & 13 & 14 & 15 \\
\hline 1 & 4 & 6 & 5 & 4 & 4 & 5 & 5 & 4 & 5 & 6 & 4 & 4 & 5 & 6 & 4 \\
\hline 2 & 3 & 2 & 2 & 3 & 3 & 2 & 2 & 3 & 2 & 2 & 3 & 3 & 3 & 2 & 2 \\
\hline 3 & 1 & 2 & 2 & 1 & 2 & 2 & 1 & 1 & 2 & 1 & 2 & 1 & 1 & 1 & 1 \\
\hline 4 & 3 & 2 & 2 & 2 & 2 & 2 & 2 & 2 & 2 & 3 & 2 & 3 & 2 & 2 & 2 \\
\hline
\end{tabular}

TABLE 4: Information on the number of outgoing passengers.

\begin{tabular}{|c|c|c|c|c|c|c|c|c|c|c|c|c|c|c|c|}
\hline \multirow{2}{*}{ Stop number } & \multicolumn{15}{|c|}{ Experiment number } \\
\hline & 1 & 2 & 3 & 4 & 5 & 6 & 7 & 8 & 9 & 10 & 11 & 12 & 13 & 14 & 15 \\
\hline 2 & 3 & 3 & 3 & 3 & 2 & 3 & 2 & 3 & 3 & 2 & 3 & 3 & 3 & 3 & 4 \\
\hline 3 & 3 & 5 & 4 & 4 & 3 & 4 & 4 & 4 & 3 & 5 & 3 & 3 & 3 & 4 & 2 \\
\hline 4 & 3 & 3 & 3 & 1 & 4 & 3 & 2 & 1 & 3 & 2 & 4 & 2 & 3 & 3 & 2 \\
\hline 5 & 2 & 1 & 1 & 2 & 2 & 1 & 2 & 2 & 2 & 3 & 1 & 3 & 2 & 1 & 1 \\
\hline
\end{tabular}

traffic flows exist between stops 1 and 2, as well as between stops 1 and 3 , stops 2 and 3 , and stops 3 and 4 .

Within the scope of another experiment, we have analyzed a route containing ten stops. In the experiment, the number of incoming passengers is a random variable with the following distribution: the number of incoming passengers on first stop takes values 4,5 , and 6 with equal probabilities $1 / 3$; on second, fourth, and eighth stops, this value is 2 or 3 with probabilities $1 / 2$; on third, seventh, and ninth stops, this value is 1 or 2 with probabilities $1 / 2$; on the sixth stop, it takes 3 , 4 with probabilities $1 / 2$; and on the fifth stop, it takes 3,4 , and 5 with probabilities $1 / 3$. Again, the tables of passenger traffic have been constructed, in this case based on 1000 numerical experiments. Tables 5 and 6 provide the information on the obtained passenger traffic flows for first 15 experiments. The acquired experimental data have been involved for evaluating the unknown passenger traffic intensities by the least-squares technique and the minimization method for the sum of absolute deviations. Clearly, both methods bring to close values of the intensities:

$$
\Lambda_{1}=\left(\begin{array}{cccccccccc}
0 & 0 & 4.61 & 0.28 & 0.06 & 0.03 & 0 & 0 & 0 & 0 \\
0 & 0 & 2.31 & 0.15 & 0.04 & 0 & 0 & 0 & 0 & 0 \\
0 & 0 & 0 & 1.07 & 0.24 & 0.15 & 0.02 & 0.02 & 0 & 0 \\
0 & 0 & 0 & 0 & 1.63 & 0.61 & 0.22 & 0.05 & 0 & 0 \\
0 & 0 & 0 & 0 & 0 & 3.2 & 0.48 & 0.32 & 0 & 0 \\
0 & 0 & 0 & 0 & 0 & 0 & 2.8 & 0.72 & 0 & 0 \\
0 & 0 & 0 & 0 & 0 & 0 & 0 & 1.42 & 0.07 & 0 \\
0 & 0 & 0 & 0 & 0 & 0 & 0 & 0 & 1.96 & 0.54 \\
0 & 0 & 0 & 0 & 0 & 0 & 0 & 0 & 0 & 1.51 \\
0 & 0 & 0 & 0 & 0 & 0 & 0 & 0 & 0 & 0
\end{array}\right),
$$


TABLE 5: Information on the number of incoming passengers.

\begin{tabular}{|c|c|c|c|c|c|c|c|c|c|c|c|c|c|c|c|}
\hline \multirow{2}{*}{ Stop number } & \multicolumn{15}{|c|}{ Experiment number } \\
\hline & 1 & 2 & 3 & 4 & 5 & 6 & 7 & 8 & 9 & 10 & 11 & 12 & 13 & 14 & 15 \\
\hline 1 & 5 & 6 & 6 & 4 & 6 & 5 & 6 & 4 & 4 & 4 & 5 & 6 & 4 & 4 & 6 \\
\hline 2 & 3 & 3 & 2 & 3 & 2 & 3 & 2 & 2 & 3 & 2 & 3 & 2 & 3 & 2 & 2 \\
\hline 3 & 2 & 1 & 1 & 2 & 2 & 1 & 1 & 2 & 2 & 2 & 2 & 2 & 2 & 2 & 1 \\
\hline 4 & 3 & 2 & 3 & 3 & 3 & 3 & 3 & 3 & 3 & 3 & 3 & 3 & 3 & 3 & 3 \\
\hline 5 & 3 & 4 & 3 & 3 & 3 & 5 & 5 & 3 & 4 & 5 & 4 & 3 & 4 & 3 & 5 \\
\hline 6 & 3 & 3 & 3 & 3 & 4 & 3 & 4 & 3 & 4 & 3 & 4 & 4 & 4 & 3 & 4 \\
\hline 7 & 1 & 1 & 2 & 1 & 2 & 1 & 1 & 1 & 1 & 2 & 2 & 2 & 1 & 1 & 1 \\
\hline 8 & 3 & 2 & 2 & 2 & 2 & 2 & 2 & 3 & 2 & 3 & 2 & 2 & 2 & 3 & 2 \\
\hline 9 & 1 & 2 & 2 & 1 & 1 & 1 & 2 & 2 & 2 & 1 & 2 & 1 & 2 & 2 & 2 \\
\hline
\end{tabular}

TABLE 6: Information on the number of outgoing passengers.

\begin{tabular}{|c|c|c|c|c|c|c|c|c|c|c|c|c|c|c|c|}
\hline \multirow{2}{*}{ Stop number } & \multicolumn{15}{|c|}{ Experiment number } \\
\hline & 1 & 2 & 3 & 4 & 5 & 6 & 7 & 8 & 9 & 10 & 11 & 12 & 13 & 14 & 15 \\
\hline 2 & 3 & 3 & 3 & 2 & 3 & 3 & 3 & 3 & 2 & 2 & 2 & 3 & 2 & 3 & 3 \\
\hline 3 & 5 & 6 & 5 & 5 & 4 & 4 & 5 & 3 & 5 & 4 & 6 & 4 & 5 & 3 & 5 \\
\hline 4 & 2 & 1 & 0 & 1 & 2 & 1 & 1 & 2 & 1 & 1 & 1 & 2 & 1 & 2 & 1 \\
\hline 5 & 1 & 2 & 3 & 1 & 4 & 2 & 3 & 2 & 2 & 2 & 3 & 4 & 2 & 2 & 3 \\
\hline 6 & 5 & 3 & 3 & 6 & 2 & 7 & 4 & 3 & 6 & 6 & 4 & 2 & 6 & 3 & 4 \\
\hline 7 & 1 & 2 & 2 & 3 & 3 & 1 & 3 & 2 & 3 & 2 & 4 & 3 & 3 & 2 & 3 \\
\hline 8 & 3 & 3 & 4 & 1 & 4 & 3 & 3 & 3 & 2 & 4 & 3 & 4 & 2 & 3 & 3 \\
\hline 9 & 3 & 1 & 2 & 2 & 2 & 1 & 2 & 3 & 2 & 3 & 1 & 2 & 2 & 3 & 2 \\
\hline 10 & 1 & 3 & 2 & 1 & 1 & 2 & 2 & 2 & 2 & 1 & 3 & 1 & 2 & 2 & 2 \\
\hline
\end{tabular}

\section{The Equilibrium Distribution Model for Passenger Traffic Flows}

In the previous section, we have suggested an evaluation procedure for passenger traffic flows among stops on a route. Now, let us analyze the equilibrium distribution of these passenger traffic flows with respect to transport companies (services).

Consider an $M$-player noncooperative game of the following form. Imagine a certain route serviced by $M$ transport companies (players). The route represents a sequence of nodes numbered from 1 to $K$ communicating via edges $(i, i+$ $1), i=1, \ldots, K-1$. Traffic on the route is from left to right. There is a given matrix of passenger traffic intensities $\lambda_{s t}$ from node $s$ to node $t, s<t$ :

$$
\Lambda=\left(\begin{array}{ccccc}
0 & \lambda_{12} & \lambda_{13} & \cdots & \lambda_{1 K} \\
0 & 0 & \lambda_{23} & \cdots & \lambda_{2 K} \\
\cdots & \cdots & \cdots & \cdots & \cdots \\
0 & 0 & \cdots & \cdots & 0
\end{array}\right) .
$$

Suppose that on edge $(i, i+1)$ of the route player $m$ transports passengers according to the exponential distribution of service time with the parameter $\mu_{i}^{m}, i=1, \ldots, K-1$.

Player $m$ assigns prices $p_{s t}^{m}$ for its services on each segment $(s, t)$ of the route $(1, K)$. Construct the strategy profile $\left\{p_{s t}^{m}, s<t, m=1, \ldots, M\right\}$ of all players. By assumption, passengers minimize their costs representing the sum of the ticket price and the expected service time (latency), thereby choosing the cheapest service. As is well-known from queueing theory (e.g., see [5]), the latency on a route having the Poisson traffic flow of intensity $\lambda$ and service intensity $\mu$ makes up $1 /(\mu-\lambda)$. It is possible to introduce more complicated cost functions, for example, the ones with weight coefficients for the service price and latency. For the sake of simplicity, our analysis gets confined to the standard sum of these costs.

Interestingly, the total passenger traffic flows on each segment of the route can be easily calculated using the given matrix of passenger traffic intensities. For instance, the total passenger traffic flow on edge $(s, s+1)$ is defined by

$$
\bar{\lambda}_{s, s+1}=\sum_{i=1}^{s} \sum_{j=s+1}^{K} \lambda_{i j}
$$

Then, the incoming flow $\lambda_{s t}$ is distributed among services, making the Poisson flows of intensities $\lambda_{s t}^{m}$, where $\sum_{m=1}^{M} \lambda_{s t}^{m}=$ $\lambda_{s t}$.

The costs of a passenger choosing service $m$ on the segment $(s, t)$ comprise the ticket price and the total latency on all edges $(i, i+1)$ belonging to $(s, t)$ :

$$
p_{s t}^{m}+\sum_{i=s}^{t-1} \frac{1}{\mu_{i}^{m}-\bar{\lambda}_{i, i+1}^{m}} .
$$


In an equilibrium, the costs of all passengers on competing routes coincide for all services. Having this in mind, find the intensities $\lambda_{s t}^{m}$ for all services $m=1, \ldots, M$ and all segments of the route $(s, t)$ :

$$
\begin{aligned}
p_{s t}^{m} & +\sum_{i=s}^{t-1} \frac{1}{\mu_{i}^{m}-\bar{\lambda}_{i, i+1}^{m}} \\
& =p_{s t}^{l}+\sum_{i=s}^{t-1} \frac{1}{\mu_{i}^{l}-\bar{\lambda}_{i, i+1}^{l}}, \quad \forall s, t: s<t, \quad l \in M .
\end{aligned}
$$

The utility of player $m \in\{1, \ldots, M\}$ can be expressed as its per-unit-time income from servicing all flows on all segments belonging to the route of this player; that is,

$$
H_{m}\left(\left\{p_{s t}^{m}\right\}\right)=\sum_{s=1}^{K-1} \sum_{t=s+1}^{K} \lambda_{s t}^{m} p_{s t}^{m}
$$

Therefore, we have completely described the game. Now, it is possible to evaluate the corresponding equilibrium. A Nash equilibrium is a price profile $\left\{P_{s t}^{m}, s<t, m=1, \ldots, M\right\}$ such that the conditions,

$H_{l}\left(p_{s t}^{l},\left\{P_{s t}^{m}, s<t, m \neq l\right\}\right) \leq H_{l}\left(\left\{P_{s t}^{m}, s<t, m=1, \ldots, M\right\}\right)$,

hold true for each player $l \in\{1, \ldots, M\}$ and any prices $p_{s t}^{l}$ assigned by it.

\section{Competition of Two Players on a Segment}

The suggested model is tested by the following simple example. There are two competing transport companies which service passengers on the segment $(A, B)$. Their service times have the exponential distribution with the parameters $\mu_{1}$ and $\mu_{2}$, respectively. Requests for services form the Poisson process with the intensity $\lambda$. Assume that $\lambda<\mu_{1}+\mu_{2}$. Players assign their service prices $p_{1}$ and $p_{2}$, respectively. Subsequently, passengers choose the cheapest service. Then, the incoming flow is decomposed into the two Poisson flows with the intensities $\lambda_{1}$ and $\lambda_{2}, \lambda_{1}+\lambda_{2}=\lambda$. The costs of a passenger choosing service $i$ constitute

$$
p_{i}+\frac{1}{\mu_{i}-\lambda_{i}}, \quad i=1,2,
$$

where $1 /\left(\mu_{i}-\lambda_{i}\right)$ is the expected duration of passenger stay in the service system. Obviously, if

$$
p_{1}+\frac{1}{\mu_{1}-\lambda}<p_{2}+\frac{1}{\mu_{2}}
$$

then all passengers choose service 1 . In the case of

$$
p_{2}+\frac{1}{\mu_{2}-\lambda}<p_{1}+\frac{1}{\mu_{1}}
$$

all passengers choose service 2 . This solution appears trivial, as far as no competition takes place. To create competition, players have to balance their prices so that the above inequalities fail. Consider the competitive case when passengers use both services.

The intensities $\lambda_{1}$ and $\lambda_{2}$ of the flows for the corresponding services can be found from the following conditions:

$$
\begin{gathered}
p_{1}+\frac{1}{\mu_{1}-\lambda_{1}}=p_{2}+\frac{1}{\mu_{2}-\lambda_{2}}, \\
\lambda_{1}+\lambda_{2}=\lambda .
\end{gathered}
$$

The utilities of the players have the following form:

$$
H_{1}\left(p_{1}, p_{2}\right)=\lambda_{1} p_{1}, \quad H_{2}\left(p_{1}, p_{2}\right)=\lambda_{2} p_{2} .
$$

For the sake of definiteness, set $\mu_{1}>\mu_{2}$. Next, fix $p_{2}$ and evaluate the best response of player 1. Apply Lagrange's method of multipliers to find the stationary points of the associated optimization problem with equality constraints. Formally, the scheme of this method admits the following representation. Maximize the function $H_{1}$ subject to the constraints (21)-(22):

$$
L_{1}=p_{1} \lambda_{1}+k_{1}\left(p_{1}+\frac{1}{\mu_{1}-\lambda_{1}}-p_{2}-\frac{1}{\mu_{2}-\lambda+\lambda_{2}}\right) .
$$

Apply the first-order necessary optimality condition:

$$
\begin{gathered}
\frac{\partial L_{1}}{\partial p_{1}}=\lambda_{1}+k_{1}=0, \quad k_{1}=-\lambda_{1}, \\
\frac{\partial L_{1}}{\partial \lambda_{1}}=p_{1}+k_{1}\left(\frac{1}{\left(\mu_{1}-\lambda_{1}\right)^{2}}+\frac{1}{\left(\mu_{2}-\lambda_{2}\right)^{2}}\right),
\end{gathered}
$$

where it appears that

$$
P_{1}=\lambda_{1}\left(\frac{1}{\left(\mu_{1}-\lambda_{1}\right)^{2}}+\frac{1}{\left(\mu_{2}-\lambda_{2}\right)^{2}}\right) .
$$

Similarly, calculate the price for player 2 :

$$
P_{2}=\lambda_{2}\left(\frac{1}{\left(\mu_{1}-\lambda_{1}\right)^{2}}+\frac{1}{\left(\mu_{2}-\lambda_{2}\right)^{2}}\right) .
$$

Formulas (26)-(27) bring to

$$
\frac{P_{1}}{\lambda_{1}}=\frac{P_{2}}{\lambda_{2}}
$$

In other words, the equilibrium intensities are proportional to the assigned prices. Therefore,

$$
\lambda_{1}=\lambda \frac{P_{1}}{P_{1}+P_{2}}, \quad \lambda_{2}=\lambda \frac{P_{2}}{P_{1}+P_{2}} .
$$

By substituting this result into (26)-(27), we arrive at the following equation:

$$
\begin{aligned}
P_{1}+P_{2}=\lambda & \frac{1}{\left(\mu_{1}-\left(P_{1} /\left(P_{1}+P_{2}\right)\right) \lambda\right)^{2}} \\
& \left.+\frac{1}{\left(\mu_{2}-\left(P_{2} /\left(P_{1}+P_{2}\right)\right) \lambda\right)^{2}}\right) .
\end{aligned}
$$


TABLE 7: The values $\left(P_{1}, P_{2}\right)$ under $\lambda=10$.

\begin{tabular}{cccccc}
\hline$\mu_{1}$ & 6 & 7 & $\mu_{2}$ & 9 & 10 \\
\hline 6 & $(10 ; 10)$ & & & & \\
7 & $(5.38 ; 5.05)$ & $(2.5 ; 2.5)$ & & & \\
8 & $(3.94 ; 3.53)$ & $(1.75 ; 1.64)$ & $(1.11 ; 1.11)$ & & \\
9 & $(3.26 ; 2.81)$ & $(1.38 ; 1.22)$ & $(0.87 ; 0.81)$ & $(0.625 ; 0.625)$ & \\
10 & $(2.86 ; 2.39)$ & $(1.18 ; 0.98)$ & $(0.73 ; 0.64)$ & $(0.52 ; 0.49)$ & $(0.4 ; 0.4)$ \\
\hline
\end{tabular}

On the other hand, the expression (21) yields

$$
P_{1}-P_{2}=\frac{1}{\mu_{2}-\left(P_{2} /\left(P_{1}+P_{2}\right)\right) \lambda}-\frac{1}{\mu_{1}-\left(P_{1} /\left(P_{1}+P_{2}\right)\right) \lambda} \text {. }
$$

The system of (30)-(31) uniquely defines the equilibrium prices $P_{1}$ and $P_{2}$. The table below gives the equilibrium prices for the fixed-intensity flow with $\lambda=10$ and different service intensities $\mu_{1}$ and $\mu_{2}$.

It is seen from Table 7 that the increasing of the service intensities decreases the equilibrium prices.

\section{Competition of $M$ Players on a Segment}

The above competition model of two servers can be easily generalized to the case of $M$ players. Imagine $M$ competitive transport companies (servers) processing requests with the exponential distribution of service time with the parameters $\mu_{1}, \mu_{2}, \ldots, \mu_{M}$, respectively. Players assign prices $p_{1}, p_{2}, \ldots, p_{M}$ for their services. Then, passengers (clients) choose the cheapest service and the incoming flow gets decomposed into $M$ Poisson flows with the intensities $\lambda_{1}, \lambda_{2}, \ldots, \lambda_{M}$, where $\lambda_{1}+\lambda_{2}+\cdots+\lambda_{M}=\lambda$. The costs of a client choosing service $i$ make up

$$
p_{i}+\frac{1}{\mu_{i}-\lambda_{i}}, \quad i=1,2, \ldots, M \text {. }
$$

And the flow intensities $\lambda_{1}, \lambda_{2}, \ldots, \lambda_{M}$ of the corresponding services can be found from the following condition:

$$
p_{1}+\frac{1}{\mu_{1}-\lambda_{1}}=p_{2}+\frac{1}{\mu_{2}-\lambda_{2}}=\cdots=p_{m}+\frac{1}{\mu_{M}-\lambda_{M}} \text {. }
$$

The utility of player $i$ takes the following form:

$$
H_{i}\left(p_{1}, p_{2}, \ldots, p_{M}\right)=\lambda_{i} p_{i}, \quad i=1, \ldots, M .
$$

Apply Lagrange's method of multipliers to evaluate the best response of player 1 . Fix $p_{i}(i=2,3, \ldots, M)$ and maximize the function $H_{1}$ subject to the constraints (33):

$$
\begin{aligned}
L_{1}= & p_{1} \lambda_{1}+\sum_{i=2}^{m} k_{i}\left(p_{1}+\frac{1}{\mu_{1}-\lambda_{1}}-p_{i}-\frac{1}{\mu_{i}-\lambda_{i}}\right) \\
& +\gamma\left(\sum_{i=1}^{m} \lambda_{i}-\lambda\right) .
\end{aligned}
$$

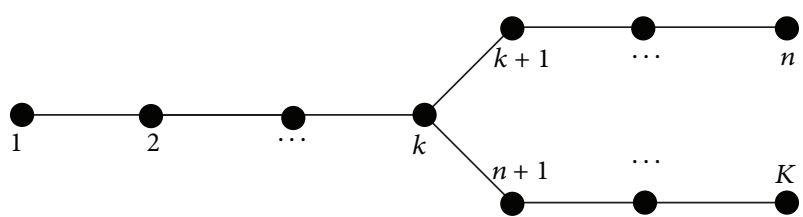

Figure 2: Competition of players on a graph.

The first-order necessary optimality conditions yield the following equations:

$$
\begin{gathered}
\frac{\partial L_{1}}{\partial p_{1}}=\lambda_{1}+\sum_{i=2}^{M} k_{i}=0, \\
\frac{\partial L_{1}}{\partial \lambda_{1}}=p_{1}+\frac{\sum_{i=2}^{M} k_{i}}{\left(\mu_{1}-\lambda_{1}\right)^{2}}+\gamma=0, \\
\frac{\partial L_{1}}{\partial \lambda_{i}}=-\frac{k_{i}}{\left(\mu_{i}-\lambda_{i}\right)^{2}}+\gamma=0, \quad i=2, \ldots, M .
\end{gathered}
$$

By repeating this procedure for each player, we construct the system of equations for defining the equilibrium prices and flow intensities:

$$
\begin{gathered}
P_{i}=\lambda_{i}\left(\frac{1}{\sum_{j=0, j \neq i}^{m}\left(\mu_{j}-\lambda_{j}\right)^{2}}+\frac{1}{\left(\mu_{i}-\lambda_{i}\right)^{2}}\right), \\
P_{i}+\frac{1}{\mu_{i}-\lambda_{i}}=P_{i+1}+\frac{1}{\mu_{i+1}-\lambda_{i+1}}, \quad i=0, \ldots, M-1, \\
\sum_{i=1}^{m} \lambda_{i}=\lambda .
\end{gathered}
$$

Table 8 gives the equilibrium prices and intensities for the fixed-intensity flow with $\lambda=10$ and fixed service intensity $\mu_{1}=6$ and various service intensities $\mu_{2}$ and $\mu_{3}$. Comparing with the case of two players, we see that the increasing of competition yields the decreasing of the equilibrium prices.

We can see from Tables 7 and 8 that the equilibrium prices decrease with the increasing of the number of players.

\section{Competition of $M$ Players on a Graph}

This model can be generalized to the case of any transportation graph. Consider for instance the competition of $M$ services on the graph which presented a linear path $\{1, \ldots, k\}$ and two paths $\{k, k+1, \ldots, n\}$ and $\{k, n+1, \ldots, K\}$ with joint node $k$ (Figure 2 ). Suppose that all incoming passengers come into a service on the first stop and then go out on the next stops of the route in the Poisson manner. Denote $\lambda_{j}$ the intensity of the flow on the path $(1, j), j=2, \ldots, K$.

Assume that each service (player) $i$ has two routs $R_{1}^{i}=$ $\{1, \ldots, k, k+1, \ldots, n\}$ and $R_{2}^{i}=\{1, \ldots, k, n+1, \ldots, K\}, i=$ $1, \ldots, M$. The service times have the exponential distribution with the parameters $\mu_{1}, \ldots, \mu_{M}$, respectively.

Players announce a price $p_{i}^{j}$ for the service on each path $(1, j), i=1, \ldots, M, j=2, \ldots, K$. The passengers choose 
TABLE 8: The values $\left(P_{1}, P_{2}, P_{3}\right)$ and $\left(\lambda_{1}, \lambda_{2}, \lambda_{3}\right)$ under $\lambda=10, \mu_{1}=6$.

\begin{tabular}{|c|c|c|c|c|c|}
\hline \multirow{2}{*}{$\mu_{2}$} & \multicolumn{5}{|c|}{$\mu_{3}$} \\
\hline & & 6 & 7 & 8 & 9 \\
\hline \multirow{2}{*}{6} & $\left(P_{1} ; P_{2}, P_{3}\right)$ & $(0.7 ; 0.7 ; 0.7)$ & & & \\
\hline & $\left(\lambda_{1} ; \lambda_{2} ; \lambda_{3}\right)$ & $(3.33 ; 3.33 ; 3.33)$ & & & \\
\hline \multirow{2}{*}{7} & $\left(P_{1} ; P_{2}, P_{3}\right)$ & $(0.54 ; 0.58 ; 0.54)$ & $(0.42 ; 0.46 ; 0.46)$ & & \\
\hline & $\left(\lambda_{1} ; \lambda_{2} ; \lambda_{3}\right)$ & $(3.12 ; 3.75,3.12)$ & $(2.92 ; 3.54 ; 3.54)$ & & \\
\hline \multirow{2}{*}{8} & $\left(P_{1} ; P_{2}, P_{3}\right)$ & $(0.43 ; 0.5 ; 0.43)$ & $(0.34 ; 0.4 ; 0.37)$ & $(0.28 ; 0.33 ; 0.33)$ & \\
\hline & $\left(\lambda_{1} ; \lambda_{2} ; \lambda_{3}\right)$ & $(2.93 ; 4.14 ; 2.93)$ & $(2.72 ; 3.93 ; 3.35)$ & $(2.52 ; 3.74 ; 3.74)$ & \\
\hline \multirow{2}{*}{9} & $\left(P_{1} ; P_{2}, P_{3}\right)$ & $(0.35 ; 0.43 ; 0.35)$ & $(0.28 ; 0.36 ; 0.31)$ & $(0.23 ; 0.3 ; 0.28)$ & $(0.19 ; 0.25 ; 0.25)$ \\
\hline & $\left(\lambda_{1} ; \lambda_{2} ; \lambda_{3}\right)$ & $(2.75 ; 4.5 ; 2.75)$ & $(2.53 ; 4.3 ; 3.17)$ & $(2.34 ; 4.1 ; 3.56)$ & $(2.14 ; 3.93 ; 3.93)$ \\
\hline
\end{tabular}

the service with minimal costs. Then, the flow $\lambda_{j}$ is split for $m$ Poisson flows with intensities $\lambda_{j}^{i}, i=1, \ldots, M: \lambda_{j}=$ $\sum_{i=1}^{M} \lambda_{j}^{i}, j=2, \ldots, K$.

The balance equations take the following form:

$$
\begin{array}{r}
p_{1}^{j}+\sum_{k=1}^{j-1} a_{k}^{(1)}-p_{i}^{j}-\sum_{k=1}^{j-1} a_{k}^{(i)}=0, \quad i=2, \ldots, M, j=2, \ldots, n, \\
p_{1}^{j}+\sum_{s=1}^{k-1} a_{s}^{(1)}+\sum_{s=n}^{j-1} a_{s}^{(1)}-p_{i}^{j}-\sum_{s=1}^{k-1} a_{s}^{(i)}-\sum_{s=n_{1}}^{j-1} a_{s}^{(i)}=0, \\
i=2, \ldots, M, \quad j=n+1, \ldots, K,
\end{array}
$$

where

$$
\begin{aligned}
& a_{s}^{(i)}=\frac{1}{\mu_{i}-\sum_{t=s+1}^{K} \lambda_{t}^{i}}, \quad i=1, \ldots, M, s=1, \ldots, k-1, \\
& a_{s}^{(i)}=\frac{1}{\mu_{i} / 2-\sum_{t=s+1}^{n} \lambda_{t}^{i}}, \quad i=1, \ldots, M, s=k, \ldots, n-1, \\
& a_{s}^{(i)}=\frac{1}{\mu_{i} / 2-\sum_{t=s+1}^{K} \lambda_{t}^{i}}, \quad i=1, \ldots, M, s=n, \ldots, K-1,
\end{aligned}
$$

which is a delay on the corresponding link $(s, s+1)$. The division of the original route into two paths leads to two different forms of the delay.

Payoff of $i$ th player is

$$
H_{i}\left(p_{1}, \ldots, p_{M}\right)=\sum_{j=2}^{K} p_{i}^{j} \lambda_{j}^{i} .
$$

Form the Lagrange function for player 1 subject to constraints (38):

$$
\begin{aligned}
L_{1}= & \sum_{j=2}^{K} p_{1}^{j} \lambda_{j}^{1}+\sum_{j=2}^{K} \gamma_{j}\left(\sum_{i=1}^{M} \lambda_{j}^{i}-\lambda_{j}\right) \\
& +\sum_{i=2}^{M} \sum_{j=2}^{n} k_{j}^{i}\left(p_{1}^{j}+\sum_{k=1}^{j-1} a_{k}^{(1)}-p_{i}^{j}-\sum_{k=1}^{j-1} a_{k}^{(i)}\right) \\
& +\sum_{i=2}^{M} \sum_{j=n+1}^{K} k_{j}^{i}\left(p_{1}^{j}+\sum_{s=1}^{k-1} a_{s}^{(1)}+\sum_{s=n}^{j-1} a_{s}^{(1)}\right. \\
& \left.-p_{i}^{j}-\sum_{s=1}^{k-1} a_{s}^{(i)}-\sum_{s=n}^{j-1} a_{s}^{(i)}\right) .
\end{aligned}
$$

By analogy, we can construct the Lagrange function for all players. The first order condition gives

$$
\begin{aligned}
& p_{i}^{s}= \sum_{l=0}^{s-2} \sum_{j=2+l}^{n} \lambda_{j}^{i}\left(\left(a_{l+1}^{(i)}\right)^{2}+\frac{1}{\sum_{t=2, t \neq i}^{m}\left(a_{l+1}^{(t)}\right)^{-2}}\right), \\
& s=2, \ldots, k, \\
& p_{i}^{s}= \sum_{l=0}^{k-2} \sum_{j=2+l}^{K} \lambda_{j}^{i}\left(\left(a_{l+1}^{(i)}\right)^{2}+\frac{1}{\sum_{t=2, t \neq i}^{M}\left(a_{l+1}^{(t)}\right)^{-2}}\right) \\
&+\sum_{l=1}^{s-k} \sum_{j=k+l}^{n} \lambda_{j}^{i}\left(\left(a_{k+l-1}^{(i)}\right)^{2}+\frac{1}{\sum_{t=2, t \neq i}^{M}\left(a_{k+l-1}^{(t)}\right)^{-2}}\right), \\
& p_{i}^{s}= \sum_{l=0}^{k-2} \sum_{j=2+l}^{K} \lambda_{j}^{i}\left(\left(a_{l+1}^{(i)}\right)^{2}+\frac{1}{\sum_{t=2, t \neq i}^{M}\left(a_{l+1}^{(t)}\right)^{-2}}\right)^{k+1, \ldots, n,}, \\
&+\sum_{l=1}^{s-n} \sum_{j=n+l}^{K} \lambda_{j}^{i}\left(\left(a_{n+l-1}^{(i)}\right)^{2}+\frac{1}{\sum_{t=2, t \neq i}^{M}\left(a_{n+l-1}^{(t)}\right)^{-2}}\right), \\
& s=n, \ldots, K,
\end{aligned}
$$


TABLE 9: The values $\left(P_{1}^{i}, P_{2}^{i}, P_{3}^{i}\right)$ and $\left(\lambda_{i}^{1}, \lambda_{i}^{2}, \lambda_{i}^{3}\right)$ under $\lambda_{2}=2, \lambda_{3}=2, \lambda_{4}=3, \lambda_{5}=3$, and $\mu_{1}=6 ; i=2,3,4$.

\begin{tabular}{llcc}
\hline$\mu_{2}$ & & $\mu_{3}$ \\
& & 6 & \\
\hline \multirow{2}{*}{} & $\left(P_{1}^{2} ; P_{2}^{2}, P_{3}^{2}\right)$ & $(0.7 ; 0.7 ; 0.7)$ & \\
& $\left(\lambda_{2}^{1} ; \lambda_{2}^{2} ; \lambda_{2}^{3}\right)$ & $(0.667 ; 0.667 ; 0.667)$ & $(0.43 ; 0.46 ; 0.46)$ \\
7 & $\left(P_{1}^{2} ; P_{2}^{2}, P_{3}^{2}\right)$ & $(0.54 ; 0.58 ; 0.54)$ & $(0.63 ; 0.68 ; 0.68)$ \\
& $\left(\lambda_{2}^{1} ; \lambda_{2}^{2} ; \lambda_{2}^{3}\right)$ & $(0.65 ; 0.7 ; 0.65)$ & $(0.34 ; 0.4 ; 0.37)$ \\
8 & $\left(P_{1}^{2} ; P_{2}^{2}, P_{3}^{2}\right)$ & $(0.43 ; 0.5 ; 0.43)$ & $(0.61 ; 0.72 ; 0.67)$ \\
\hline \multirow{2}{*}{6} & $\left(\lambda_{2}^{1} ; \lambda_{2}^{2} ; \lambda_{2}^{3}\right)$ & $(0.63 ; 0.74 ; 0.63)$ & \\
& $\left(P_{1}^{3} ; P_{2}^{3}, P_{3}^{3}\right)$ & $(1.06 ; 1.06 ; 1.06)$ & $(0.66 ; 0.72 ; 0.72)$ \\
7 & $\left(\lambda_{3}^{1} ; \lambda_{3}^{2} ; \lambda_{3}^{3}\right)$ & $(0.667 ; 0.667 ; 0.667)$ & $(0.63 ; 0.68 ; 0.68)$ \\
& $\left(P_{1}^{3} ; P_{2}^{3}, P_{3}^{3}\right)$ & $(0.83 ; 0.9 ; 0.83)$ & $(0.53 ; 0.64 ; 0.59)$ \\
& $\left(\lambda_{2}^{1} ; \lambda_{2}^{2} ; \lambda_{2}^{3}\right)$ & $(0.65 ; 0.7 ; 0.65)$ & $(0.62 ; 0.71 ; 0.67)$ \\
\hline \multirow{2}{*}{6} & $\left(P_{1}^{3} ; P_{2}^{3}, P_{3}^{3}\right)$ & $(0.66 ; 0.78 ; 0.66)$ & \\
& $\left(\lambda_{3}^{1} ; \lambda_{3}^{2} ; \lambda_{3}^{3}\right)$ & $(0.64 ; 0.72 ; 0.64)$ & $(0.6 ; 0.7 ; 0.7)$ \\
\hline 7 & $\left(P_{1}^{4} ; P_{2}^{4}, P_{3}^{4}\right)$ & $(1.44 ; 1.44 ; 1.44)$ & $(0.9 ; 1.01 ; 1.01)$ \\
& $\left(\lambda_{4}^{1} ; \lambda_{4}^{2} ; \lambda_{4}^{3}\right)$ & $(1 ; 1 ; 1)$ & $(0.82 ; 1.09 ; 1.09)$ \\
& $\left(P_{1}^{4} ; P_{2}^{4}, P_{3}^{4}\right)$ & $(1.13 ; 1.25 ; 1.13)$ & $(0.73 ; 0.91 ; 0.83)$ \\
& $\left(\lambda_{4}^{1} ; \lambda_{4}^{2} ; \lambda_{4}^{3}\right)$ & $(0.91 ; 1.18 ; 0.91)$ & $(0.75 ; 1.25 ; 1)$ \\
\hline
\end{tabular}

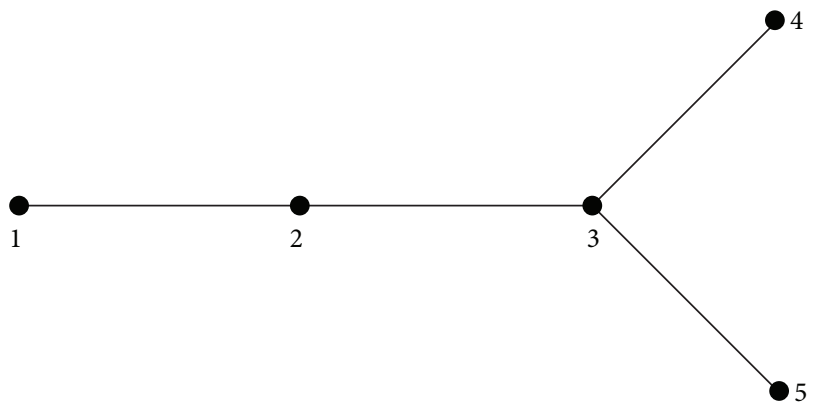

FIGURE 3: Competition of players on a graph.

for $i=1,2, \ldots, M$, where $a_{s}^{(i)}$ is determined by (39). Systems (38)-(42) determine the equilibrium prices and flow intensities in the game.

In Table 9, the equilibrium prices and flow intensities are calculated for three players on the route with five stops (see Figure 3) and parameters $\lambda_{2}=2, \lambda_{3}=2, \lambda_{4}=3, \lambda_{5}=3$, and $\mu_{1}=6$. The prices $P^{4}$ and $P^{5}$ are equal according to the symmetry. We can see from Table 9 that increasing of the intensity of the service yields the decreasing of the service price in equilibrium. Notice, when the intensity of the service of one player is larger, then the other passengers prefer to use this service ignoring the larger price.

\section{Conclusion}

This paper has suggested a statistical calculation model for the intensity of passenger traffic flows among stops. Using a series of natural experiments, we have illustrated the evaluation procedure of passenger traffic flows among stops. Passenger traffic flows have been calculated through the leastsquares technique and the minimization method for the sum of absolute deviations. Note that the proposed evaluation procedure for passenger traffic flows admits easy realization in practice. For instance, in several countries, passengers register their railway and underground tickets in special automata while entering and leaving a vehicle. Moreover, in cities passengers often enter buses in the first place and also register their tickets in special automata. Passengers leave buses through other doors, where additional automata can be placed too.

The obtained information on passenger traffic flows can be adopted to model the distribution of passengers among transport facilities. In this work, we have proposed employing the idea of Hotelling's duopoly. By assumption, most passengers are rational. They compare their individual costs from utilizing different services and minimize them. The costs of passengers have been comprehended as the sum of the service price and service time. We have presented a gametheoretic model of price formation, which allows assessing the distribution of passengers with respect to transport companies (servers). This can be useful while estimating the characteristics of urban transport networks.

\section{Conflict of Interests}

The authors declare that there is no conflict of interests regarding the publication of this paper. 


\section{Acknowledgments}

This research is supported by Russian Fund for Basic Research (project 13-01-00033-a), the Division of Mathematical Sciences of Russian Academy of Sciences, and Saint-Petersburg State University (project no. 9.38.245.2014).

\section{References}

[1] A. G. Wilson, "A statistical theory of spatial distribution models," Transportation Research, vol. 1, pp. 253-270, 1967.

[2] A. G. Wilson, Entropy in Urban and Regional Modelling, Pion, London, UK, 1970.

[3] A. G. Wilson, "A family of spatial interaction models and associated developments," Environment and Planning A, vol. 3, pp. 255-282, 1971.

[4] N. R. Draper and H. Smith, Applied Regression Analysis, Wiley, 3rd edition, 1998.

[5] H. A. Taha, Operation Research: An Introduction, Prentice Hall, 8th edition, 2006.

[6] Y. Sheffy, Urban Transportation Networks, Prentice Hall, Englewood Cliffs, NJ, USA, 1985.

[7] J. G. Wardrop, "Some theoretical aspects of road traffic research," ICE Proceedings: Engineering Divisions, vol. 1, no. 3, pp. 325-378, 1952.

[8] H. Spiess and M. Florian, "Optimal strategies: a new assignment model for transit networks," Transportation Research, Part B, vol. 23, no. 2, pp. 83-102, 1989.

[9] H. Yang and H.-J. Huang, "The multi-class, multi-criteria traffic network equilibrium and systems optimum problem," Transportation Research Part B: Methodological, vol. 38, no. 1, pp. 1-15, 2004.

[10] P. Naor, “The regulation of queue size by levying tolls," Econometrica, vol. 37, no. 1, pp. 15-24, 1969.

[11] I. Luski, "On partial equilibrium in a queuing system with two servers," The Review of Economic Studies, vol. 43, no. 3, pp. 519$525,1976$.

[12] D. Levhari and I. Luski, "Duopoly pricing and waiting lines," European Economic Review, vol. 11, no. 1, pp. 17-35, 1978.

[13] H. Chen and Y.-W. Wan, "Capacity competition of make-toorder firms," Operations Research Letters, vol. 33, no. 2, pp. 187194, 2005.

[14] H. Hotelling, "Stability in competition," The Economic Journal, vol. 39, pp. 41-57, 1929.

[15] R. Hassin and M. Haviv, To Queue or Not to Queue. Equilibrium Behavior in Queueing Systems, Springer, 2003.

[16] W. H. Greene, Econometric Analysis, Prentice Hall, 7th edition, 2012.

[17] C. Davino, M. Furno, and D. Vistocco, Quantile Regression: Theory and Applications, John Wiley \& Sons, New York, NY, USA, 2013. 


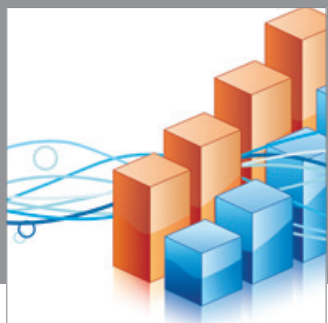

Advances in

Operations Research

mansans

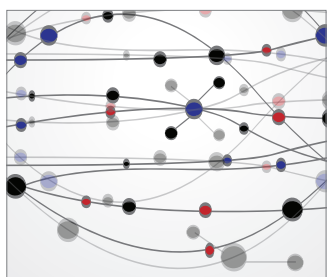

The Scientific World Journal
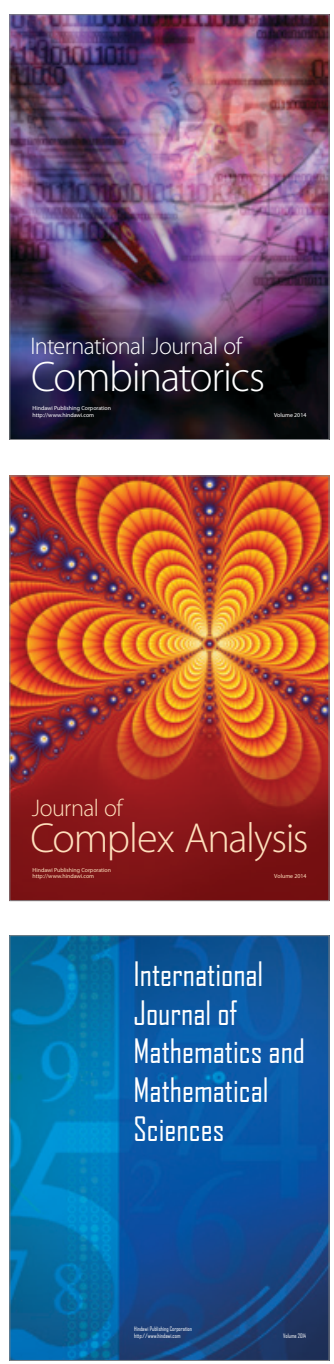
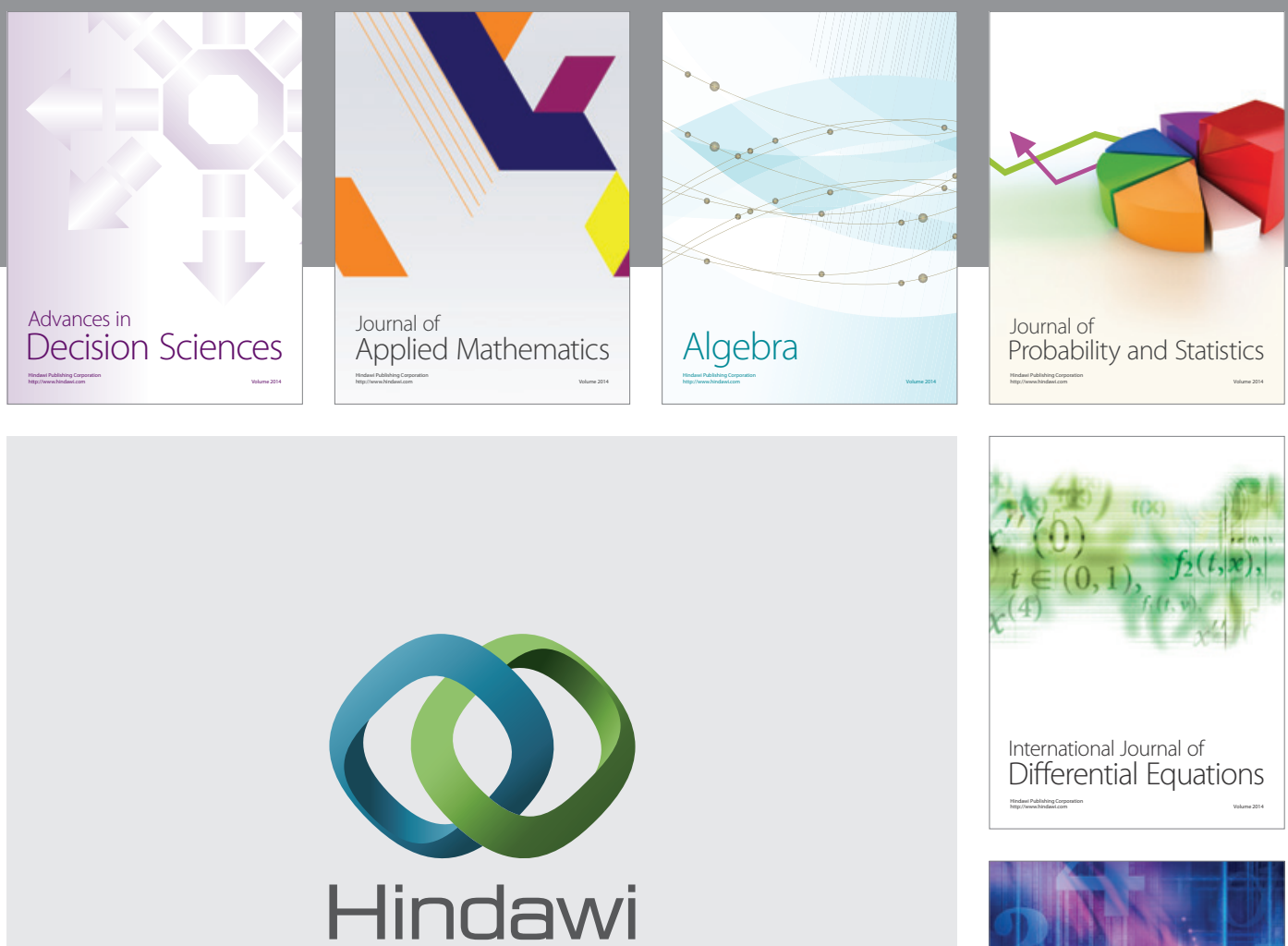

Submit your manuscripts at http://www.hindawi.com
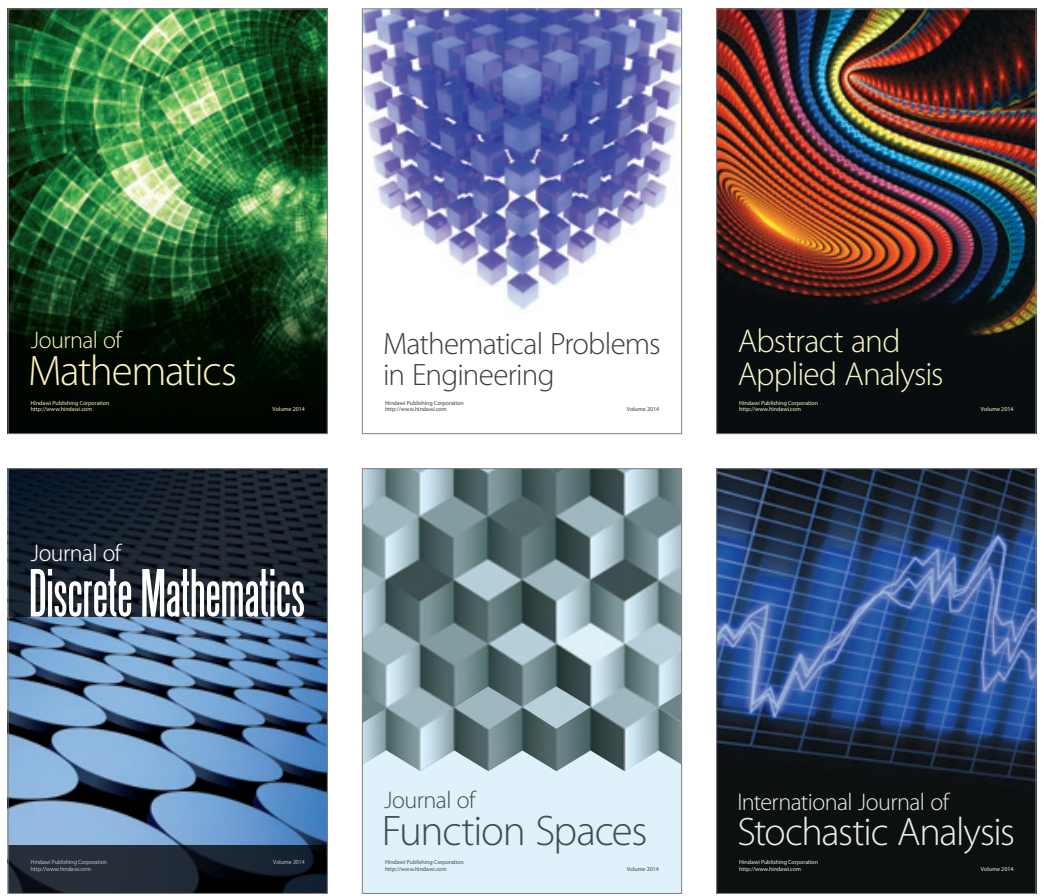

Journal of

Function Spaces

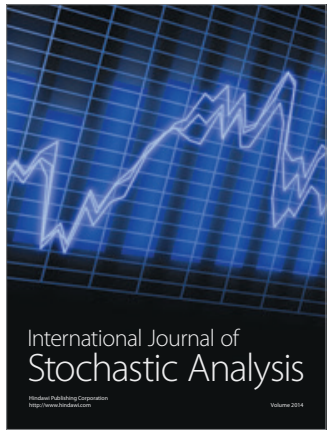

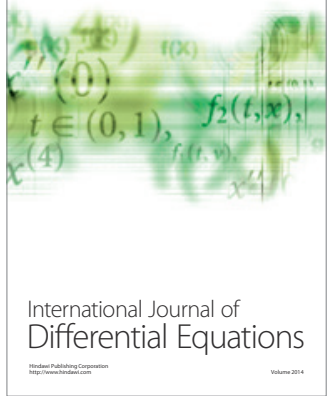
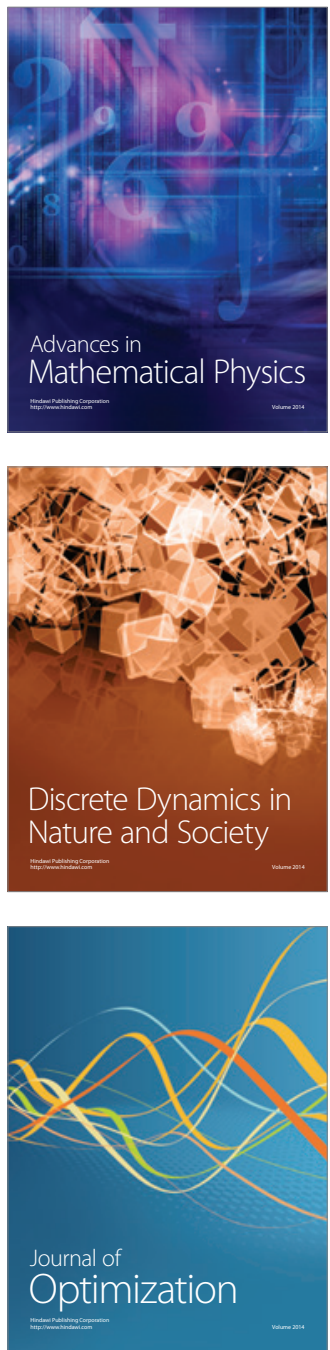